\title{
Mechanisms of $\mathrm{NH}_{3}$ and $\mathrm{NO}_{2}$ detection in
}

\section{carbon-nanotube-based sensors: An $a b$ initio investigation}

\author{
Yan Li*, Miroslav Hodak, Wenchang Lu, and J. Bernholc ${ }^{*}$ \\ Center for High Performance Simulation and Department of Physics, North Carolina State \\ University, Raleigh, NC 27695-7518
}

\begin{abstract}
The mechanisms of $\mathrm{NH}_{3}$ and $\mathrm{NO}_{2}$ detection by single-walled carbon nanotube-based devices are investigated by $a b$ initio calculations and the non-equilibrium Greens function (NEGF) methodology. While both $\mathrm{NH}_{3}$ and $\mathrm{NO}_{2}$ can physisorb to a pristine carbon nanotube, we show that their adsorption only results in small current changes through the device. For a carbon nanotube (CNT) attached to gold nanowire leads, the most sensitive detection site is at the CNT near the CNT-Au contact, where chemisorption occurs. The resulting change in electron transmission and low-bias current can lead to over 30\% sensitivity. While both $\mathrm{NH}_{3}$ and $\mathrm{NO}_{2}$ can also chemisorb at the Au electrodes, their adsorption results in only a small change in electron density of the leads, and thus a small effect on current. In order to enhance the detection sensitivity, it is thus beneficial to mask the electrodes to prevent chemisorption. Furthermore, the length of the pure CNT segment does not strongly affect the relative sensitivity. Our results suggest that a short-CNT device with exposed contact regions and masked electrodes would have the greatest sensitivity.
\end{abstract}

\footnotetext{
* Corresponding authors: Y. Li (E-mail: yli26@ncsu.edu) or J. Bernholc (E-mail: bernholc@ncsu.edu)
} 


\section{Introduction}

Single-walled carbon nanotubes [1] (SWCNTs) are promising materials and building blocks for future nanoelectronics [2-4], due to their large surface area to volume ratios, unique electronic properties, as well as high chemical and mechanical stabilities. Experiments have demonstrated that CNTs can be used as chemical sensors to detect small concentrations of gas molecules with high sensitivity $[5,6]$. The CNT-based sensors could thus impact a diverse range of human activities: from environmental monitoring, safety control of chemical processes and space missions, to agricultural and medical diagnostic applications [7]. Traditional gas sensors, based on semiconducting metal oxides, usually operate at high temperatures $\left(250-700{ }^{\circ} \mathrm{C}\right)$, because the gas molecules need to overcome an energy barrier to react with the sensing material [8]. SWCNT devices, however, have exhibited substantial electrical conductivity changes when exposed to $\mathrm{NH}_{3}$ and $\mathrm{NO}_{2}$ molecules at room temperature [6], and thus are able to act as room-temperature electrical sensors.

Since the initial discoveries, much progress has been made in improving the performance of CNT-based gas sensors by functionalizing CNTs with polymers or metal nanoparticles [9-14]. However, the underlying sensing mechanism still remains disputed. Several possibilities have been proposed: One is an indirect interaction of the gas molecules with either the hydroxyl group on $\mathrm{SiO}_{2}$ substrate [6], or the pre-adsorbed water layers around CNT in the ambient [15]. Another is direct charge transfer from adsorbed gas molecules to an individual CNT [16], or to CNT bundles with the gas molecules adsorbed strongly at interstitial sites [17]. Other alternatives include spontaneous dissociation of the gas molecules by defects containing pre-dissociated oxygen from the environment [18], and Schottky barrier modulation at the electrode-CNT contact due to presence of polarized gas molecules [19]. 
To optimize the performance of CNT based sensors for practical applications, it is important to understand the origin of the sensing signals. Does the signal arise from the central region of CNT or from the metal-CNT contacts? Experimentalists studied this issue by selectively passivating different parts of sensor devices. After passivating the metal-CNT contact with a thermally evaporated silicon oxide [20], the sensitivity to $\mathrm{NH}_{3}$ was similar to that of the normal unpassivated devices, thus suggesting that $\mathrm{NH}_{3}$ mainly interacts with the CNT in the central region. However, an experiment from a different group using poly(methyl methacrylate) (PMMA) for the passivation layers, found good sensitivities to $\mathrm{NH}_{3}$ and $\mathrm{NO}_{2}$ for both center-passivated and contact-passivated devices, indicating that both the central CNT region and the metal-CNT contacts participate in the detection process [21]. Interestingly, when another group used the same PMMA passivation material, the results were inconsistent with the previous studies. In this case, after the metal-CNT contacts were covered with PMMA, the device became insensitive to $\mathrm{NO}_{2}$, leading to the suggestion that $\mathrm{NO}_{2}$ mainly interacts with the metal-CNT contact region [22]. The inconsistencies in these studies could be due to two reasons: First, the permeable passivation materials used in the experiments could still allow gas molecules to diffuse through and interact with the passivated regions. Second, since the experiments were carried out in ambient air, air molecules could interact with the device, making the identification of the sensing mechanism(s) more difficult. To resolve these issues, Zhang and coworkers investigated $\mathrm{NH}_{3}$ sensing using selective $\mathrm{Si}_{3} \mathrm{~N}_{4}$ passivation and dry air as the background gas [23]. They observed that the sensing signal from the metal-CNT contacts is dominant while the signal from the midsection of CNT is negligible.

These experimental works provide great insights to the sensing mechanism of $\mathrm{NH}_{3}$ and $\mathrm{NO}_{2}$ detection. However, since the contact-passivated regions are usually hundreds of 
nanometers long, the passivation layers actually cover both the CNT and the electrode near the metal-CNT interface, precluding detailed atomistic understanding: does the sensing signal originates from the molecule with the metal or the CNT? Moreover, it is unknown how such interactions affect the electronic structure and transport properties of sensor devices. So far, most of the existing theoretical calculations $[6,16,17,24]$ address only the properties of pure CNTs, and show that the adsorbed gas molecules are weakly bound with a small charge transfer (about $0.1 e^{-}$per $\mathrm{NO}_{2}$ molecule and $0.04 e^{-}$per $\mathrm{NH}_{3}$ molecule). It is not clear whether the small charge transfer between the CNT and absorbed molecules causes any measurable effect on electron transport. The existing theoretical studies $[25,26]$ of the effect of metal contacts used classical transistor models to investigate Schottky barrier modulation in the presence of gas molecules, without explicit electron transport calculations.

In this paper, we investigate from first-principles the sensing abilities of several potential sensor configurations, with the gas molecule adsorbed at different positions relative to the nanotube: (i) the molecule physisorbed to CNT in the central part of the device; (ii) the molecule chemisorbed to CNT near the metal-CNT contact, and (iii) the molecule chemisorbed to the metal electrode. We examine and compare the structural and electronic transport properties of these sensing configurations using density functional theory (DFT) simulations combined with non-equilibrium Green's function (NEGF) $[27,28]$ methods. To our knowledge, this is the first quantum transport calculation that investigates the role of metal contacts in the detection of $\mathrm{NH}_{3}$ and $\mathrm{NO}_{2}$ molecules. Previous DFT studies mainly focused on the properties of pure nanotubes rather than the electrode-nanotube junctions. Our results show that a large response signal occurs only when $\mathrm{NH}_{3}$ or $\mathrm{NO}_{2}$ are chemisorbed to $\mathrm{CNT}$ at the metal-CNT contact. In the other cases, there are nearly negligible changes in electronic transport after 
adsorption of the molecule(s). Our results provide a clear atomic understanding of the sensing mechanism for gaseous $\mathrm{NH}_{3}$ and $\mathrm{NO}_{2}$ detection by room temperature CNT-based sensors.

\section{Methods and Simulations}

Our simulations employ a CNT/Au geometry that is widely used in experiments, see Figure 1, where an $(8,0)$ semiconducting SWCNT is sandwiched between two Au nanowires. The gold electrodes are connected to the nanotube through the (100) surface and consist of four periodic unit cells (8 layers) in the transport direction on each side. We also include 4 gold layers on each side of the nanotube as buffer layers in the self-consistent "device" region to allow for self-consistent screening and charge redistribution at the electrode/nanotube interfaces. The electron transport is along the axis of the CNT (x-axis), and a $12 \AA$ vacuum region is added in the $\mathrm{y}$ and $\mathrm{z}$ directions to eliminate interactions between periodic images.

The device configurations are optimized using the Real-space MultiGrid (RMG) code [29]. The convergence criterion for structural optimizations is that all atomic forces are less than $0.05 \mathrm{eV} / \mathrm{A}$. For exchange-correlation functionals, DFT-D [30] or vdW-DF [31] are used to account for the weak interactions between the molecule and the CNT in the physisorption cases, while the PBE functional [32] is used in the chemisorption cases. The Quantum Espresso code [33] is used to relax the physisorbed structures with the van der Waals corrections. The Vanderbilt ultrasoft pseudopotentials are employed in all of our calculations. A grid spacing of 0.3 bohr is used in real-space calculations and a 35 Ry kinetic energy cutoff for wavefunctions is used in Quantum Espresso.

The charge transfer in physisorption cases is calculated using the Löwdin population analysis method [34,35], which partitions the electron density by projecting the wave functions onto an orthogonal set of basis functions localized on atoms. It should be noted that 
determination of charge transfer in $a b$ initio calculations is non-unique and different methods lead to somewhat different quantitative values as shown in Ref. 36, which compared two definitions of charge transfer: one based on the shift of Fermi energy resulting from adatom adsorption and the other on integration of charge density in real space.

The quantum transport calculations are carried out using the non-equilibrium Green's function (NEGF) method $[27,28]$ expanded in the basis of variationally optimized localized orbitals [37-39]. The transport system is divided into three parts, the left electrode, the right electrode, and the center device region between the electrodes. We use six orbitals per atom for C, $\mathrm{H}, \mathrm{N}, \mathrm{O}$ and ten orbitals for Au with a cutoff radius of 9.5 Bohr. The localized orbitals are separately optimized for the three parts. The charge density and the potentials for the whole device region are calculated self-consistently using the NEGF method at the source-drain bias of $100 \mathrm{mV}$, with energy broadening of $0.5 \mathrm{meV}$ used for stability. The transmission spectrum is then calculated by the Landauer formula [40 ]

$$
T(E)=\operatorname{Tr}\left[\Gamma_{L} G_{D}^{R} \Gamma_{R} G_{D}^{A}\right]
$$

and the current by

$$
I(V)=\frac{2 e^{2}}{h} \int_{-\infty}^{\infty} T(E)\left[f\left(E-\mu_{L}\right)-f\left(E-\mu_{R}\right)\right] d E
$$

where $\mathrm{f}$ is the Fermi-Dirac distribution function, and $\mu_{L}\left(\mu_{R}\right)$ are the chemical potentials of the left (right) electrodes, respectively. All the calculations are carried out on the Blue Waters supercomputer at National Center for Supercomputing Applications and on the Titan Supercomputer at the National Center for Computational Sciences at ORNL using up to 400 CPU cores. 


\section{Results and discussion}

We describe the results for $\mathrm{NH}_{3}$ and $\mathrm{NO}_{2}$ in separate subsections. In each case, the sensing mechanism is identified and separately discussed. For the case of $\mathrm{NH} 3$, we also show that while the current decreases with an increase of nanotube length, the length of the nanotube does not strongly affect detection sensitivity.

\subsection{Sensing mechanism of $\mathrm{NH}_{3}$}

We first investigate the detection sensitivity of several ammonia adsorption configurations: (i) direct adsorption of $\mathrm{NH} 3$ to $\mathrm{CNT}$ in the midsection of the device. (ii) $\mathrm{NH} 3$ chemisorption to CNT near the metal-CNT contact, (iii) NH3 chemisorption to the metal electrode. For the last case, we position gas molecules on gold atoms in the buffer layers between the electrode and the nanotube. This represents the experimental case of gas molecules chemisorbing to the electrodes near the contact region. The left panel of Figure 1 shows the optimized geometries of the device regions, together with the distances between the ammonia and the adsorption site. Note that (a), (b), (c) in Figure 1 represent three different adsorption sites. In each case, only one molecule is adsorbed on the device. 

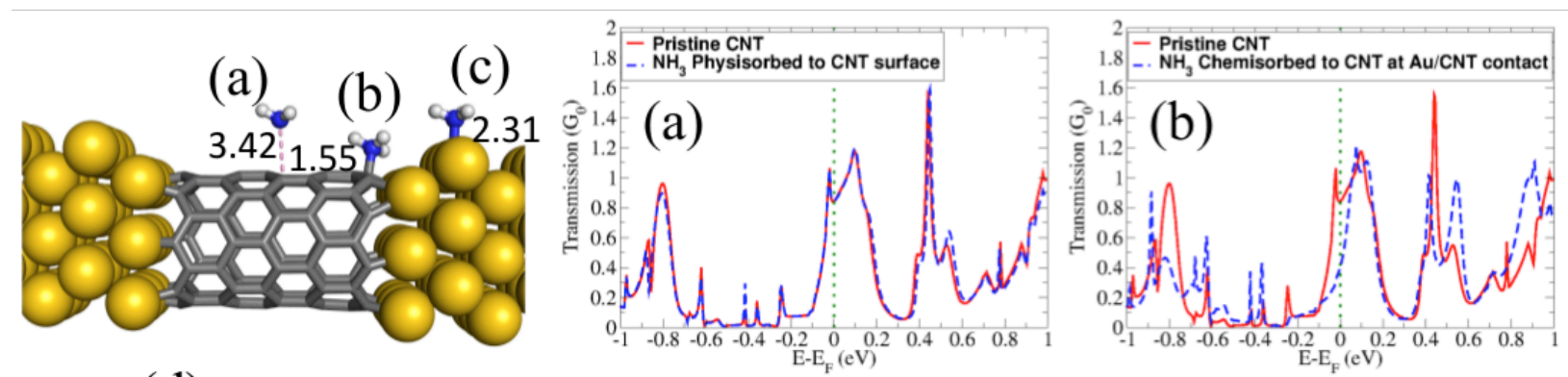

(d) 80
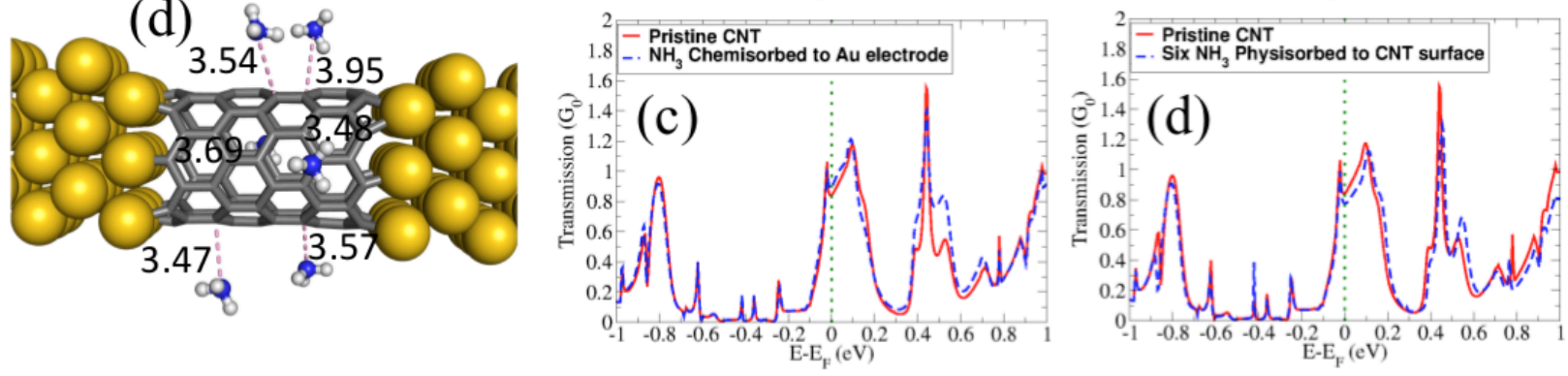

Figure 1. Optimized structures (left panel) and transmission spectra (right panel) at 100 $\mathrm{mV}$ source-drain bias of semiconducting $(8,0)$ CNT-based devices with adsorbed $\mathrm{NH}_{3}$ : (a) $\mathrm{NH}_{3}$ physisorbed to CNT, (b) $\mathrm{NH}_{3}$ chemisorbed to CNT near the Au-CNT contact, (c) $\mathrm{NH}_{3}$ chemisorbed to the Au electrode, and (d) six $\mathrm{NH}_{3}$ molecules physisorbed to CNT. In the left panel, the distances between $\mathrm{NH}_{3}$ and the adsorption sites are shown in units of $\AA$, and the dotted lines visualize the physisorption. The colors of $\mathrm{Au}, \mathrm{C}, \mathrm{N}$, and $\mathrm{H}$ are gold, grey, blue, and white, respectively. In the right panel, the transmission spectrum of a pristine CNT is shown in red for reference in each plot.

Table 1 lists the corresponding binding energies of each adsorption configuration, which are all positive and thus suggest the feasibility of adsorption. When $\mathrm{NH}_{3}$ adsorbs at the central part of CNT, only physisorption with a long NH3-CNT distance of (3.42 $\AA$ ) is found to be stable, which agrees with the results of earlier work [16]. When physisorbed, $\mathrm{NH} 3$ donates 0.11 electrons to the CNT, which is also consistent with previous results $[6,16,17,24]$. However, when $\mathrm{NH}_{3}$ is adsorbed to the $\mathrm{CNT}$ at the metal-CNT contact, chemisorption occurs with a bond length of $1.55 \AA$. Note that the binding energy $(0.097 \mathrm{eV})$ is relatively small in this case. This 
is because one C-Au bond between the CNT and the electrode gets weaker by $0.533 \mathrm{eV}$ during the formation of the C-N bond between this particular $\mathrm{C}$ atom and the ammonia molecule. Since the new C-N bond lowers the energy by $0.63 \mathrm{eV}$, the total energy is lowered by 0.097 $\mathrm{eV}$. The most favorable adsorption site is for the direct chemisorption on the Au electrode. In this case, the N-Au bond length is $2.31 \AA$ and the binding energy is $0.646 \mathrm{eV}$.

Table 1. Comparison between different adsorption configurations of $\mathrm{NH}_{3}$ in Figure 1.

\begin{tabular}{|c|c|c|c|c|c|}
\hline Adsorption configuration & $\begin{array}{l}\text { Distance } \\
(\AA)\end{array}$ & $\begin{array}{c}E_{\text {binding }} \\
(\mathrm{eV})\end{array}$ & $\begin{array}{l}\text { Conductance } \\
\qquad\left(\mathrm{G}_{0}\right)\end{array}$ & $\begin{array}{c}\text { Current } \\
(\mu \mathrm{A})\end{array}$ & Sensitivity \\
\hline Pristine CNT & & & 0.841 & 6.83 & \\
\hline (a) $\mathrm{NH}_{3}$ physisorbed to center part of CNT & 3.42 & 0.043 & 0.834 & 6.83 & $0 \%$ \\
\hline (b) $\mathrm{NH}_{3}$ chemisorbed to CNT near contact & 1.55 & 0.097 & 0.399 & 3.76 & $-44.9 \%$ \\
\hline (c) $\mathrm{NH}_{3}$ chemisorbed to the Au electrode & 2.31 & 0.646 & 0.892 & 7.14 & $+4.5 \%$ \\
\hline (d) $\mathrm{Six} \mathrm{NH}_{3}$ physisorbed to $\mathrm{CNT}$ & 3.47-3.95 & $0.013-0.052$ & 0.776 & 6.21 & $-9.1 \%$ \\
\hline
\end{tabular}

Quantum transport properties are calculated for the optimized geometries using the NEGF method. A source-drain bias of $100 \mathrm{mV}$ is chosen as in most of the experiments. The calculated electron transmission spectra are shown in the right panel of Figure 1. For each of the transmission plots in Figure 1, we also display the transmission spectrum of the pristine CNT in red. The current is calculated by integrating each transmission spectrum over a 100 $\mathrm{mV}$ bias window centered at the Fermi energy. Therefore, transmission near the Fermi energy affects the electronic transport properties the most. Since the nanotube is quite short, electron transfer from the Au electrodes makes the CNT highly n-doped. As a result, the Fermi energy of the Au lead is near the LUMO state of the CNT. Furthermore, because of the strong interaction between the lead and the CNT, the LUMO peak is broadened dramatically. Comparing the 
transmissions of adsorbed-ammonia and pristine CNT devices, the most significant differences in the transmission spectra occur in the case when the ammonia molecule chemisorbs to CNT at the CNT-Au contact, see Figure 1b. We also list the calculated conductances and currents for each binding configuration in Table 1, together with the sensitivity, defined in this paper as the relative change in current with respect to that of the pristine CNT device. The physisorbed ammonia only changes the conductance at the Fermi level from $0.841 \mathrm{G}_{0}$ to $0.834 \mathrm{G}_{0}$, where $\mathrm{G}_{0}=2 \mathrm{e}^{2} / \mathrm{h}$ denotes the conductance quantum. Chemisorption to the Au electrode increases the conductance by a few percent, mainly because the gold buffer layers have many conducting levels and the adsorption of ammonia does not significantly alter the plurality of the conducting levels of the gold layers. However, chemisorption to CNT near the Au-CNT contact results in a large decrease in conductance by $52.6 \%$.

At the experimentally used bias of $100 \mathrm{mV}$, the current is mainly determined by the conductance at the Fermi energy. Therefore, the current sensitivity is similar to the conductance sensitivity discussed above. Adsorption of a single ammonia to CNT near the Au-CNT interface decreases the current by $44.9 \%$ in our calculations, while other adsorption configurations leave the current largely unaffected. Therefore, interaction between the ammonia and the CNT near the metal-CNT contacts dominates the sensing performance and leads to high sensitivity in both conductance and current.

However, as discussed above, the most favorable adsorption site is at the electrode, while the site with the largest detection sensitivity is at the CNT near the metal-CNT contact. Therefore, to improve the sensing performance of the device, one should mask the electrodes so that most of the gas molecules adsorb to the CNT near the metal-CNT contact. Our result that ammonia binds weakly to the midsection of the CNT and only donates a small amount of 
charge, thereby not yielding significant changes in both the conductance and the current, is consistent with previous theoretical calculations $[16,17,24]$. To see whether a higher concentration of ammonia molecules improves the sensitivity in the central part of the CNT, we also consider adsorption of six ammonia molecules in the middle of the CNT. The optimized structure and the transmission spectrum are displayed in Figure 1. The adsorption distances to CNT vary slightly from $3.47 \AA$ to $3.95 \AA$, depending on the orientation of each molecule relative to the CNT surface. The binding energies for each molecule range between $0.013-0.052 \mathrm{eV}$, as listed in Table 1. Together, the six ammonia molecules donate 0.67 electrons to the CNT, which is about six times the electron transfer amount from a single ammonia molecule. Nevertheless, similarly to the case of one ammonia physisorption in the midsection of CNT, the changes in conductance and current are quite small.

In a real sensor device, the nanotube is usually much longer than that discussed above. However, our simulation of a sensor configuration with twice long CNT segment shows that the sensing mechanism is still the same as in the short-CNT device. The left panel in Figure 2 displays the different adsorption configurations and the ammonia-CNT distances, and Table 2 lists the corresponding binding energies. Similarly to the case with a short CNT segment, the most energetically favorable adsorption site is at the gold electrode and the most sensitive site is at the CNT near the contact. When the molecule is adsorbed in the middle of the CNT, only physisorption is stable. The ammonia molecules donate the same amounts of charge to the CNT as in the case with the shorter CNT: 0.11 electrons when only one $\mathrm{NH}_{3}$ is physisorbed and 0.67 electrons when six $\mathrm{NH}_{3}$ molecules are physisorbed. 

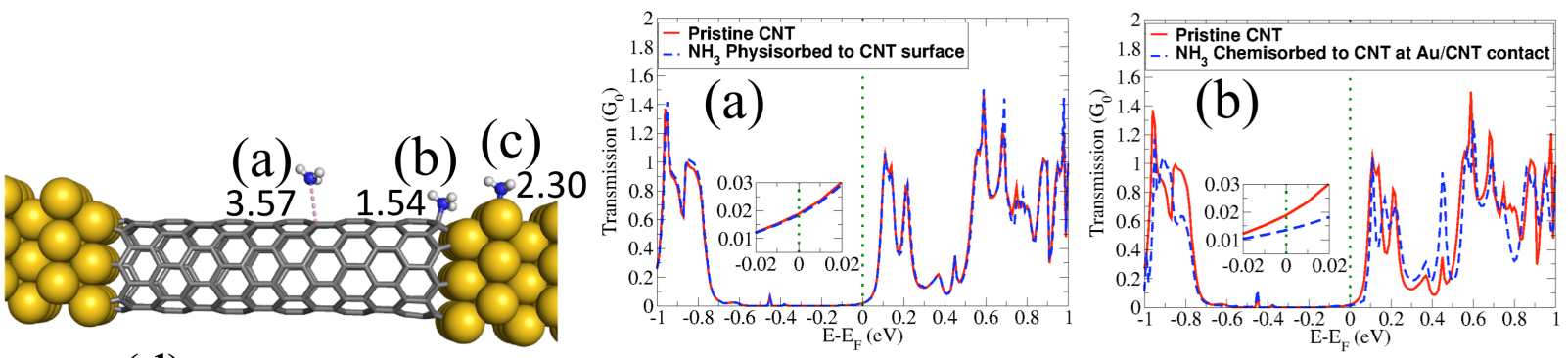

(d)
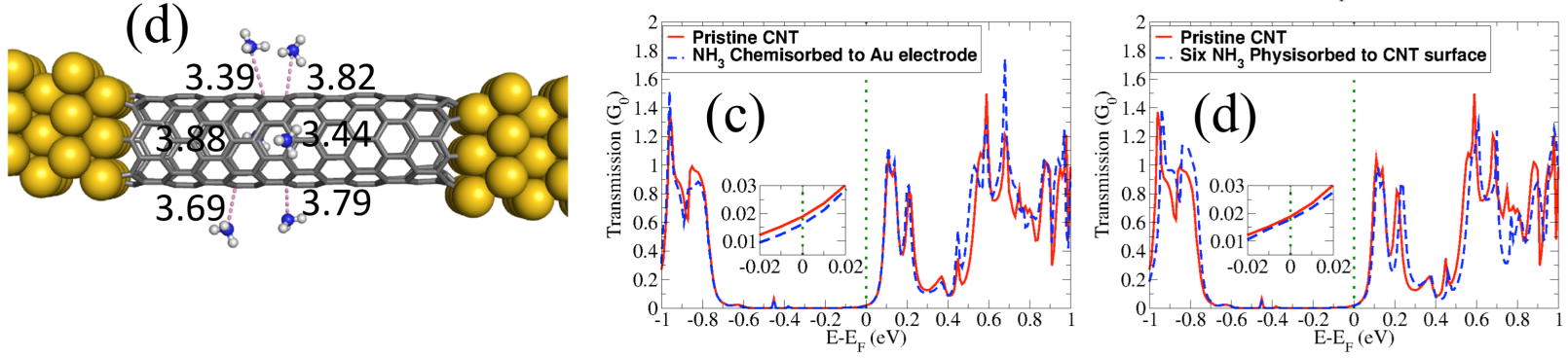

Figure 2. Optimized structures (left panel) and their transmission spectra (right panel) at 100 $\mathrm{mV}$ source-drain bias on two times as long CNT-based device as in Figure 1: (a) $\mathrm{NH}_{3}$ physisorbed to $\mathrm{CNT}$, (b) $\mathrm{NH}_{3}$ chemisorbed to $\mathrm{CNT}$ near the Au-CNT contact, (c) $\mathrm{NH}_{3}$ chemisorbed to the Au electrode, and (d) six $\mathrm{NH}_{3}$ molecules physisorbed to the $\mathrm{CNT}$ surface. In the left panel, the distances between the $\mathrm{NH}_{3}$ and the adsorption sites are shown in $\AA$, and the dotted lines visualize the physisorption. The right panel shows the transmission spectra, with that of the pristine CNT device shown in red for reference. Insets: close-ups of the transmission spectra around the Fermi energy.

The right panel of Figure 2 shows the transmission spectra of the long-CNT devices under $100 \mathrm{mV}$ source-drain bias. Comparing with the transmission in Figure 1, the LUMO peak positions are shifted away from the Fermi energy. Since we have the same Au-CNT contact, the charge transfer from Au to CNT is about the same for both short- and long-CNT device, but the contact induced doping concentration is smaller for the long-CNT devices. The transmission peaks are less broadened for the long-CNT device, due to the weaker coupling between the 
$\mathrm{Au}$ leads and the LUMO of CNT. As a result, the conductance at the Fermi level strongly decreases.

\begin{tabular}{lccccc}
\hline Adsorption configuration & Distance & $\begin{array}{c}\mathrm{E}_{\text {binding }} \\
(\AA)\end{array}$ & $\begin{array}{c}\text { Conductance } \\
(\mathrm{eV})\end{array}$ & $\begin{array}{c}\text { Current } \\
\left(\mathrm{G}_{0}\right)\end{array}$ & $\begin{array}{c}\text { Sensitivity } \\
(\mu \mathrm{A})\end{array}$ \\
\hline Pristine CNT & & & 0.0188 & 0.225 \\
(a) $\mathrm{NH}_{3}$ physisorbed to center part of CNT & 3.57 & 0.041 & 0.0184 & 0.218 & $-3.1 \%$ \\
(b) $\mathrm{NH}_{3}$ chemisorbed to CNT near contact & 1.54 & 0.151 & 0.0135 & 0.135 & $-40.0 \%$ \\
(c) $\mathrm{NH}_{3}$ chemisorbed to the Au electrode & 2.30 & 0.647 & 0.0161 & 0.22 & $-2.2 \%$ \\
(d) $\mathrm{Six} \mathrm{NH}_{3}$ physisorbed to CNT & $3.39-3.88$ & $0.020-0.046$ & 0.0178 & 0.20 & $-11.1 \%$
\end{tabular}

Table 2. Comparison between different adsorption configurations of $\mathrm{NH}_{3}$ to the long $\mathrm{CNT}$ device displayed in Figure 2.

The conductances and currents for each adsorption case are listed in Table 2. Although their absolute values are one order of magnitude smaller than those in Table 1, the sensing mechanism is still the same as for the short-CNT device. At $100 \mathrm{mV}$ bias, there is a $40.0 \%$ decrease in current when an ammonia chemisorbs to CNT near the Au-CNT contact, while the currents in other adsorption configurations are only weakly affected. Hence, our main conclusion remains unaltered: the interaction between ammonia and CNT at the metal-CNT contacts plays the dominant role in the sensing performance.

\subsection{Sensing mechanism of $\mathrm{NO}_{2}$}

Similarly, we also investigate the electrical detection of several $\mathrm{NO}_{2}$ adsorption configurations: (i) Direct adsorption of $\mathrm{NO}_{2}$ to the midsection of CNT through two $\mathrm{O}$ atoms. (ii) Direct adsorption 
of $\mathrm{NO}_{2}$ to the midsection of CNT through only one $\mathrm{O}$ atom. In these two cases, the long $\mathrm{NO}_{2}$-CNT distances correspond to physisorption, as was also found in earlier work [16]. (iii) $\mathrm{NO}_{2}$ chemisorbed to CNT near the metal-CNT contact, and (iv) $\mathrm{NO}_{2}$ chemisorbed to the metal electrode. The left panel of Figure 3 shows the optimized geometries for the device regions, together with the distances between the $\mathrm{NO}_{2}$ and the adsorption site. Table 3 lists the binding energies of each adsorption configuration, which are all positive and sufficiently large to be viable at room temperature. We find that physisorption occurs when $\mathrm{NO}_{2}$ binds to a pure CNT far away from the leads, bridged by either one or two oxygen atoms. When $\mathrm{NO}_{2}$ physisorbs through two oxygen atoms, it withdraws 0.069 electrons from the CNT. When it physisorbs through only one oxygen atom, it withdraws 0.045 electrons. The small amounts of charge transfer are consistent with previous studies $[6,16,17,24]$. However, $\mathrm{NO}_{2}$ can chemisorb to the $\mathrm{Au}$ electrode through two oxygen atoms. In this case, the two O-Au bond lengths are 2.28 and $2.33 \AA$, respectively, and the binding energy is $1.42 \mathrm{eV}$. Nevertheless, the most favorable adsorption site is at $\mathrm{CNT}$ near the Au-CNT interface. $\mathrm{NO}_{2}$ chemisorbs there with the binding energy of $1.75 \mathrm{eV}$ and the $\mathrm{N}-\mathrm{C}$ bond length is $1.43 \AA$.

We show the transmission spectra under $100 \mathrm{mV}$ source-drain bias, calculated using the NEGF formalism, in the right panel of Figure 3. The transmission near the Fermi level is the most significantly affected when $\mathrm{NO}_{2}$ chemisorbs to CNT near a Au-CNT contact. 
(a)

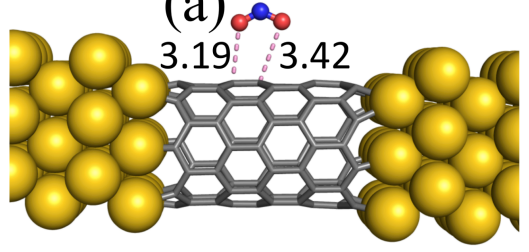

(b) \& (c) (d)

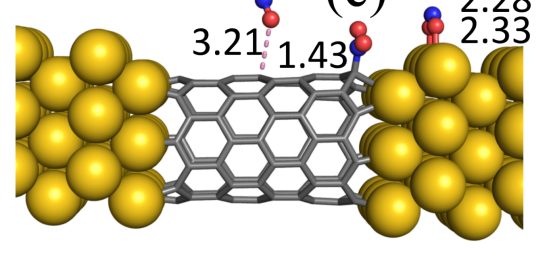

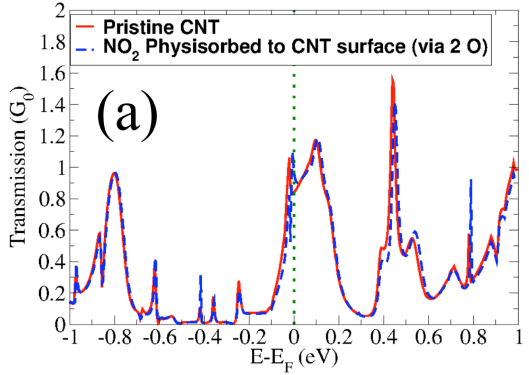
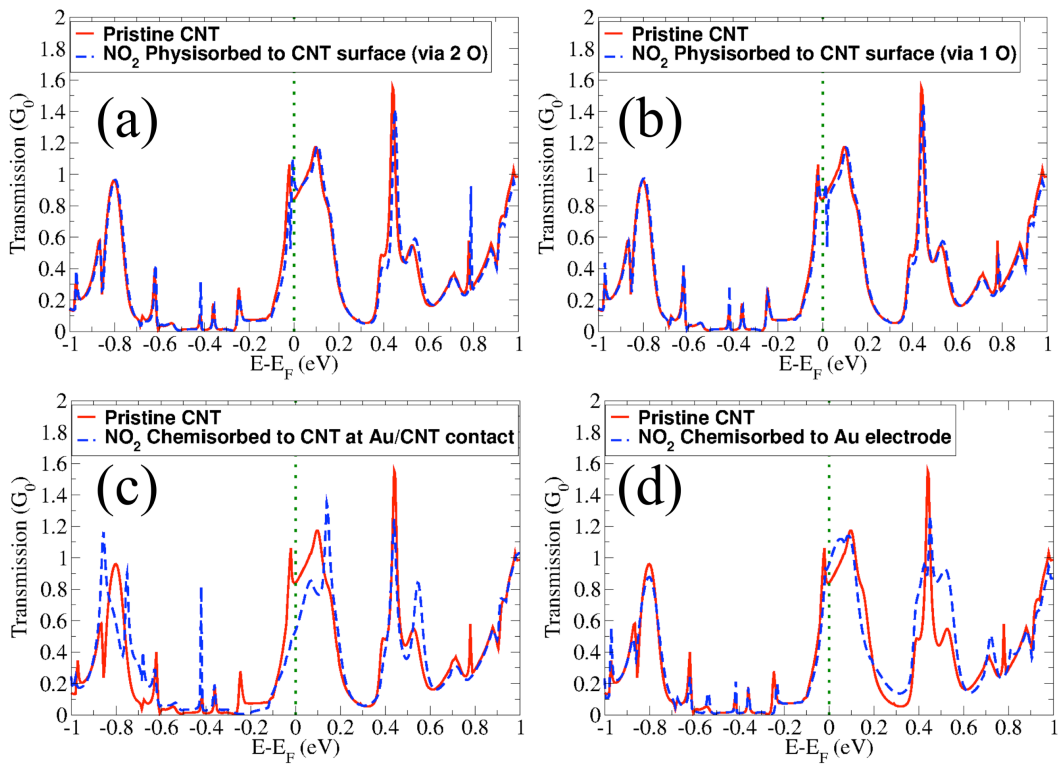

Figure 3. Optimized structures (left panel) of $\mathrm{NO}_{2}$ adsorption and their transmission spectra (right panel) at $100 \mathrm{mV}$ source-drain bias on a CNT-based device: (a) $\mathrm{NO}_{2}$ physisorbed to CNT through two $\mathrm{O}$ atoms, (b) $\mathrm{NO}_{2}$ physisorbed to CNT through one $\mathrm{O}$ atom, (c) $\mathrm{NO}_{2}$ chemisorbed to CNT near the Au-CNT contact, and (d) $\mathrm{NO}_{2}$ chemisorbed to a Au electrode. In the left panel, the distances between the $\mathrm{NO}_{2}$ and the adsorption sites are shown in $\AA$, and the dotted lines visualize physisorption. The colors of $\mathrm{Au}, \mathrm{C}, \mathrm{N}$, and $\mathrm{O}$ are gold, grey, blue, and red, respectively. In the right panel, the transmission spectrum of pristine CNT is shown in red for reference in each plot.

We also list the conductances and currents for each adsorption configuration in Table 3. It shows that the most significant change in conductance, a decrease by $36.5 \%$, occurs when $\mathrm{NO}_{2}$ chemisorbs to CNT near a Au-CNT contact, while the changes are much smaller in other cases. For example, the conductance increases by only $13.7 \%$ when $\mathrm{NO}_{2}$ chemisorbs to the $\mathrm{Au}$ electrode, and increases by $16.5 \%$ when $\mathrm{NO}_{2}$ physisorbs to CNT far from the contacts through two oxygen atoms. 
Table 3. Comparison between different adsorption configurations of $\mathrm{NO}_{2}$ in Figure 3.

\begin{tabular}{lccccc}
\hline Adsorption configuration & Distance & $\begin{array}{c}E_{\text {binding }} \\
(\AA)\end{array}$ & $\begin{array}{c}\text { Conductance } \\
(\mathrm{eV})\end{array}$ & $\begin{array}{c}\text { Current } \\
\left(\mathrm{G}_{0}\right)\end{array}$ & $\begin{array}{c}\text { Sensitivity } \\
(\mu \mathrm{A})\end{array}$ \\
\hline Pristine CNT & & & 0.841 & 6.83 \\
(a) $\mathrm{NO}_{2}$ physisorbed to CNT through 2 O & $3.19,3.42$ & 0.261 & 0.980 & 6.39 & $-6.4 \%$ \\
(b) $\mathrm{NO}_{2}$ physisorbed to CNT through 1 O & 3.21 & 0.228 & 0.850 & 6.35 & $-7.0 \%$ \\
(c) $\mathrm{NO}_{2}$ chemisorbed to CNT near contact & 1.43 & 1.75 & 0.534 & 4.41 & $-35.4 \%$ \\
(d) $\mathrm{NO}_{2}$ chemisorbed to Au contact & $2.28,2.33$ & 1.42 & 0.956 & 7.11 & $+4.1 \%$ \\
\hline
\end{tabular}

At $100 \mathrm{mV}$ source-drain bias, the most substantial change in current is a $35.4 \%$ decrease when $\mathrm{NO}_{2}$ chemisorbs to $\mathrm{CNT}$ near a contact, while physisorption on pristine CNT reduces the current by only 6-7\%. Interestingly, chemisorption on the $\mathrm{Au}$ nanowire results in a $4 \%$ increase. However, practical electrodes would be much wider, with much larger density of electrons at the Fermi level, thus diminishing this signal greatly. In sum, detection of a small amount $\mathrm{NO}_{2}$ is dominated by adsorption near the CNT-metal contact, while high concentration of physisorbed $\mathrm{NO}_{2}$ can also contribute to the signal. During the detection process, the interaction between the $\mathrm{NO}_{2}$ molecule and $\mathrm{CNT}$ at the metal-CNT contacts dominates the sensing performance, leading to high sensitivities in both the conductance and the current. Since the binding energy of $\mathrm{NO}_{2}$ is greater near the CNT-metal contact than at the electrode, the masking of the electrode is less necessary than in the case of ammonia, but some binding to the electrode may still occur, which would diminish the sensitivity.

In the above, we evaluated the sensing mechanism of $\mathrm{NH}_{3}$ and $\mathrm{NO}_{2}$ detection using the semiconducting $(8,0)$ CNT as a model system. If a metallic CNT is used instead, there would be 
a much larger base current across the device, which would not be affected as much by gating due to adsorption of one or a few gas molecules, whose effect would be screened by the free charge. Hence, we do not expect metallic nanotubes to be highly effective as the active parts of molecular sensors. For semiconducting nanotubes, their sensing response should mainly depend on their band gap, which determines the amount of screening charge available at room temperature, rather than their chirality. Since the band gap of a semiconducting nanotube inversely depends on its diameter, if a semiconducting CNT with a larger diameter is used, larger currents but smaller sensitivities would be expected. Given that charge transfer mainly depends on local geometry, we expect that the dominant sensing mechanism will remain the same for small and medium diameter nanotubes, with the most sensitive adsorption site being on the CNT near its contact with the electrode.

\section{Conclusions}

We have systematically investigated the sensing mechanisms of CNT-based devices for the detection of $\mathrm{NH}_{3}$ and $\mathrm{NO}_{2}$ using first-principles calculations and the non-equilibrium Green's function method. We examined the electrical detection of different adsorption configurations, with the molecules adsorbed in different positions relative to the metal electrodes. To the best of our knowledge, these are the first quantum transport calculations that consider the detection of $\mathrm{NH}_{3}$ and $\mathrm{NO}_{2}$ not only by the pristine CNT segments, but also near the contacts and at the electrodes.

Our results indicate that the dominant sensing mechanism in the low concentration regime is the interaction between the molecule and the CNT at the metal-CNT contacts. In this case, the transmission spectrum near the Fermi energy shows the most significant change compared to that of the pristine CNT. This change is reflected in the low-bias-voltage current, 
resulting in substantial detection sensitivity. In all other cases, when a molecule adsorbs to either a pristine CNT or to the electrode, the changes in both conductance and current are much smaller, resulting in low or even negligible sensitivity.

Our calculations provide a clear atomistic understanding of the sensing mechanism for gaseous $\mathrm{NH}_{3}$ and $\mathrm{NO}_{2}$ detection at room temperature. Interactions between the gas molecule and CNT near the electrode-CNT contact result in chemisorption, which affects the electron distribution near the contact and therefore the transmission. However, for ammonia molecules we find that the most favorable adsorption site is at the electrode. Therefore, to improve the sensing performance, one should mask the electrode to prevent ammonia binding.

For $\mathrm{NO}_{2}$, the most favorable adsorption site and the most sensitive site are the same, i.e., at the CNT near the electrode-CNT contact. At the low concentration limit, most of the $\mathrm{NO}_{2}$ molecules will adsorb there. However, the binding energies of competing sites are also significant and may result in binding at room temperature. Masking of the electrodes may thus be advisable even in this case.

\section{Acknowledgments}

We would like to thank Prof. P. Barbara for correspondence and bringing her $\mathrm{NO}_{2}$ results to our attention.

This work was supported by DOE DE-FG02-98ER45685. Petascale code development was funded by NSF ACI-1339844. The supercomputer time was provided by NSF grant OCI-1036215 at the National Center for Supercomputing Applications (NSF OCI-0725070 and ACI-1238993) and by DOE at the National Center for Computational Sciences at ORNL. 


\section{REFERENCES}

1. Iijima, S. Helical microtubules of graphitic carbon. Nature 1991, 354, 56-58.

2. Tans, S. J.; Devoret, M. H.; Dai, H.; Thess, A.; Smalley, R. E.; Georliga, L.; Dekker, C. Individual single-wall carbon nanotubes as quantum wires. Nature 1997, 386, 474-477.

3. Baughman, R. H.; Zakhidov, A. A.; de Heer, W. A. Carbon nanotubes-the route toward applications. Science 2002, 297, 787-792.

4. Tans, S. J.; Verschueren, A. R.; Dekker, C. Room-temperature transistor based on a single carbon nanotube. Nature 1998, 393, 49-52.

5. Collins, P. G.; Bradley, K.; Ishigami, M.; Zettl, A. Extreme oxygen sensitivity of electronic properties of carbon nanotubes. Science 2000, 287, 1801-1804.

6. Kong, J.; Franklin, N. R.; Zhou, C.; Chapline, M. G.; Peng, S.; Cho, K.; Dai, H. Nanotube molecular wires as chemical sensors. Science 2000, 287, 622-625.

7. Watson, J.; Ihokura, K. Gas-sensing materials. MRS Bull. 1999, 24, 14-17.

8. Fine, G. F.; Cavanagh, L. M.; Afonja, A.; Binions, R. Metal oxide semi-conductor gas sensors in environmental monitoring. Sensors 2010, 10, 5469-5502.

9. Qi, P.; Vermesh, O.; Grecu, M.; Javey, A.; Wang, Q.; Dai, H.; Peng, S.; Cho, K. Toward large arrays of multiplex functionalized carbon nanotube sensors for highly sensitive and selective molecular detection. Nano Lett. 2003, 3, 347-351.

10. An, K. H.; Jeong, S. Y.; Hwang, H. R.; Lee, Y. H. Enhanced sensitivity of a gas sensor incorporating single-walled carbon nanotube-polypyrrole nanocomposites. Adv. Mater. 2004, 16, 1005-1009. 
11. Bekyarova, E.; Davis, M.; Burch, T.; Itkis, M.; Zhao, B.; Sunshine, S.; Haddon, R. Chemically functionalized single-walled carbon nanotubes as ammonia sensors. J. Phys. Chem. B 2004, 108, 19717-19720.

12. Zhang, T.; Mubeen, S.; Bekyarova, E.; Yoo, B. Y.; Haddon, R. C.; Myung, N. V.; Deshusses, M. A. Poly (m-aminobenzene sulfonic acid) functionalized single-walled car- bon nanotubes based gas sensor. Nanotechnology 2007, 18, 165504.

13. Kong, J.; Chapline, M. G.; Dai, H. Functionalized c arbon nanotubes for molecular hydrogen sensors. Adv. Mater. 2001, 13, 1384-1386.

14. Star, A.; Joshi, V.; Skarupo, S.; Thomas, D.; Gabriel, J.-C. P. Gas sensor array based on metal-decorated carbon nanotubes. J. Phys. Chem. B 2006, 110, 21014-21020.

15. Bradley, K.; Gabriel, J.-C. P.; Briman, M.; Star, A.; Grüner, G. Charge transfer from ammonia physisorbed on nanotubes. Phys. Rev. Lett. 2003, 91, 218301.

16. Chang, H.; Lee, J. D.; Lee, S. M.; Lee, Y. H. Adsorption of $\mathrm{NH}_{3}$ and $\mathrm{NO} 2$ molecules on carbon nanotubes. Appl. Phys. Lett. 2001, 79, 3863-3865.

17. Zhao, J.; Buldum, A.; Han, J.; Lu, J. P. Gas molecule adsorption in carbon nanotubes and nanotube bundles. Nanotechnology 2002, 13, 195.

18. Andzelm, J.; Govind, N.; Maiti, A. Nanotube-based gas sensors-Role of structural defects. Chem. Phys. Lett. 2006, 421, 58-62.

19. Yamada, T. Equivalent circuit model for carbon nanotube Schottky barrier: Influence of neutral polarized gas molecules. Appl. Phys. Lett. 2006, 88, 083106.

20. Bradley, K.; Gabriel, J.-C. P.; Star, A.; Grüner, G. Short-channel effects in contact-passivated nanotube chemical sensors. Appl. Phys. Lett. 2003, 83, 3821-3823. 
21. Liu, X.; Luo, Z.; Han, S.; Tang, T.; Zhang, D.; Zhou, C. Band engineering of carbon nanotube field-effect transistors via selected area chemical gating. Appl. Phys. Lett. 2005, 86, 243501.

22. Zhang, J.; Boyd, A.; Tselev, A.; Paranjape, M.; Barbara, P. Mechanism of NO2 detection in carbon nanotube field effect transistor chemical sensors. Appl. Phys. Lett. 2006, 88, 123112.

23. Peng, N.; Zhang, Q.; Chow, C. L.; Tan, O. K.; Marzari, N. Sensing mechanisms for carbon nanotube based $\mathrm{NH}_{3}$ gas detection. Nano Lett. 2009, 9, 1626-1630.

24. Bauschlicher Jr, C. W.; Ricca, A. Binding of $\mathrm{NH}_{3}$ to graphite and to a $(9,0)$ carbon nanotube. Phys. Rev. B 2004, 70, 115409.

25. Heinze, S.; Tersoff, J.; Martel, R.; Derycke, V.; Appenzeller, J.; Avouris, P. Carbon nanotubes as Schottky barrier transistors. Phys. Rev. Lett. 2002, 89, 106801.

26. Yamada, T. Modeling of carbon nanotube Schottky barrier modulation under oxidizing conditions. Phys. Rev. B 2004, 69, 125408.

27. Larade, B.; Taylor, J.; Mehrez, H.; Guo, H. Conductance, I-V curves, and negative differential resistance of carbon atomic wires. Phys. Rev. B 2001, 64, 075420.

28. Brandbyge, M.; Mozos, J.-L.; Ordejon, P.; Taylor, J.; Stokbro, K. Density-functional method for nonequilibrium electron transport. Phys. Rev. B 2002, 65, 165401.

29. Briggs, E.; Sullivan, D.; Bernholc, J. Real-space multigrid-based approach to large-scale electronic structure calculations. Phys. Rev. B 1996, 54, 14362.

30. Grimme, S.; Antony, J.; Ehrlich, S.; Krieg, H. A consistent and accurate ab initio parametrization of density functional dispersion correction (DFT-D) for the 94 elements H-Pu. J. Chem. Phys. 2010, 132, 154104. 
31. Dion, M.; Rydberg, H.; Schroder, E.; Langreth, D. C.; Lundqvist, B. I. Van der Waals density functional for general geometries. Phys. Rev. Lett. 2004, 92, 246401.

32. Perdew, J. P.; Burke, K.; Ernzerhof, M. Generalized gradient approximation made simple. Phys. Rev. Lett. 1996, 77, 3865.

33. Giannozzi, P.; Baroni, S.; Bonini, N.; Calandra, M.; Car, R.; Cavazzoni, C.; Ceresoli, D.; Chiarotti, G. L.; Cococcioni, M.; Dabo, I. et al. QUANTUM ESPRESSO: a modular and open-source software project for quantum simulations of materials. J. Phys.: Condens Matter 2009, 21, 395502.

34. Löwdin, P.-O. On the Non-Orthogonality Problem Connected with the Use of Atomic Wave Functions in the Theory of Molecules and Crystals. J Chem Phys 1950, 18, 365.

35. Löwdin, P.-O. On the orthogonality problem. Adv Quantum Chem 1970, 5, 185.

36. Chan, K. T.; Neaton, J. B.; Cohen, M. L. First-principles study of metal-adatom adsorption on graphene. Phys. Rev. B 2008, 77, 235430.

37. Fattebert, J.-L.; Bernholc, J. Towards grid-based O (N) density-functional theory methods: Optimized nonorthogonal orbitals and multigrid acceleration. Phys. Rev. B 2000, 62, 1713.

38. Lu, W.; Meunier, V.; Bernholc, J. Nonequilibrium quantum transport properties of organic molecules on silicon. Phys. Rev. Lett. 2005, 95, 206805.

39. Buogiorno Nardelli, M.; Fattebert, J.-L.; Bernholc, J. O(N) real-space method for ab initio quantum transport calculations: Application to carbon nanotube-metal contacts. Phys. Rev. B 2001, 64, 245423.

40. Landauer, R. Spatial variation of currents and fields due to localized scatterers in metallic conduction. IBM J. Res. Dev. 1957, 1, 223-231. 